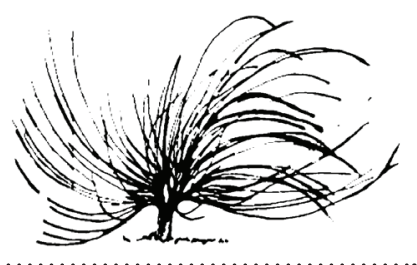

\title{
Análisis de la gestión administrativa de centros educativos costarricenses: Percepción del colectivo docente y la dirección
}

\author{
Virginia Cerdas Montano ${ }^{1}$ \\ Universidad Nacional \\ Heredia, Costa Rica \\ norma.cerdas.montano@una.cr
}

Nancy Torres Vitoria
Universidad Nacional
Heredia, Costa Rica
nancy.torres.vitoria@una.cr

\author{
José Antonio García Martínez $z^{2}$ \\ Universidad Nacional \\ Heredia, Costa Rica \\ jose.garcia.martinez@una.cr \\ Manuel Arturo Fallas Vargas ${ }^{4}$ \\ Universidad Nacional \\ Heredia, Costa Rica \\ manuel.fallas.vargas@una.cr
}

\section{(묘요}

Recibido: 10 de noviembre de 2016-Aprobado: 3 de octubre de 2017

http://dx.doi.org/10.15359/rep.12-2.5

1 Doctora en Educación con especialidad en Mediación Pedagógica. Especialidad en Gestión Educativa con énfasis en liderazgo, ejerce como académica e investigadora en la División de Educación para el Trabajo (CIDE) de la Universidad Nacional, Costa Rica.

2 Máster en Educación y Tecnologías con énfasis en Investigación por la Universidad Obierta de Catalunya. Ejerce como docente e investigador en la División de Educación para el Trabajo (CIDE) de la Universidad Nacional, Costa Rica.

3 Doctora en Educación con especialidad en Mediación Pedagógica. Historiadora y curriculista, ejerce como académica e investigadora en la División de Educación Rural y la División de Educación para el Trabajo (CIDE) de la Universidad Nacional, Costa Rica.

4 Doctor en Sexualidad y Relaciones Interpersonales por la Universidad de Salamanca, Magíster en Psicopedagogía y Licenciado en Ciencias de la Educación con énfasis en Orientación. Actualmente ejerce como docente e investigador en la División de Educación para el trabajo (CIDE) de la Universidad Nacional, Costa Rica. 


\section{Resumen}

Los centros educativos, como organizaciones complejas y dinámicas, se caracterizan por los procesos sociales que los constituyen para el logro de metas y objetivos comunes. En este sentido, la gestión administrativa tiene el reto de favorecer alternativas pertinentes a cada contexto y que den respuesta a las necesidades e intereses de la comunidad educativa, al mismo tiempo que responda a la estructura administrativa del sistema en el que está inmerso. El presente estudio tiene el objetivo de analizar la percepción del colectivo docente y la dirección sobre aspectos relacionados con la gestión administrativa. La metodología utilizada para el cumplimiento de los objetivos propuestos se plantea desde un enfoque mixto, específicamente desde una perspectiva cuantitativa a través de un diseño no experimental y transversal, mientras que la cualitativa se basa en un método fenomenológico. La recolección de datos se realiza con un cuestionario ad hoc aplicado a 192 docentes y entrevistas semiestructuradas a seis responsables de la dirección de centros educativos públicos costarricenses. Los resultados muestran que existe cierto desconocimiento por el colectivo docente sobre las funciones inherentes a la gestión administrativa. Igualmente existen aspectos relevantes en relación con las directrices emanadas por el Ministerio de Educación Pública en respuesta a las necesidades del contexto, la infraestructura y los recursos presupuestarios destinados por el Estado para el apoyo a la docencia, así como la participación docente en actividades propias del centro.

Palabras clave: administración educativa, gestión educativa, organización de centros educativos, educación.

\section{Abstract}

Educational institutions, as complex and dynamic organizations, are characterized by the social processes that lead them to the accomplishment of goals and common objectives. Regarding this matter, administrative management has the challenge of favoring pertinent alternatives for every context offering answers to the 
necessities and interests of the educational community and, at the same time, of being responsive for the administrative structure of the system of which it is a part. This study has the objective of analyzing both the teaching staff and the management's perception about administrative management. The methodology used for the accomplishment of the objectives arises from a mixed approach, specifically from a quantitative perspective through a non-experimental transversal design, while the qualitative approach is based on a phenomenological method. Data recollection was done whit an ad hoc questionnaire applied to 192 teachers and semi structured interviews conducted to six Costa Rican public school managers. Results show that there is some lake of knowledge by the teaching staff on the functions inherent in the administrative management; likewise, there are relevant aspects regarding the guidelines emanated from the Ministry of Public Education in response to the needs of the context, infrastructure and economic resources destined by the Costa Rican State as means to support teaching as well as teachers participation in the school activities.

Keywords: educational administration, administrative management, organization of schools, education.

\section{Introducción}

La Universidad Nacional de Costa Rica fortalece sus procesos académicos desde la extensión, docencia e investigación. Respondiendo a estos procesos se desarrolla el proyecto de investigación denominado "Tendencias y Desafíos de la Administración Costarricense", en el cual se plantean objetivos en relación con dos dimensiones: lo administrativo y lo curricular. En este artículo se pretende aportar específicamente a la dimensión administrativa de los centros educativos.

En este sentido se asume el centro educativo como una organización compleja y dinámica, estas características son propias de procesos sociales donde las personas que la conforman deben interactuar para el logro de metas y objetivos comunes. Partiendo de estas características, se requiere de procesos de gestión administrativos que favorezcan alternativas pertinentes a cada contexto y que puedan responder a las necesidades e intereses de la comunidad educativa, al 
mismo tiempo que responda a la estructura administrativa del sistema en el que está inmerso.

En la gestión de centros educativos se vuelve relevante saber cómo las personas que participan del proceso están percibiendo la integralidad de este, ya que en esta medida su participación estará direccionada al logro de objetivos y metas comunes.

El objetivo de esta investigación es analizar la percepción del colectivo docente y la dirección sobre aspectos relacionados con la gestión administrativa de los centros educativos a partir de las disposiciones (políticas educativas) del Ministerio de Educación Pública (MEP), para incidir en los factores limitantes desde el proceso de formación superior y de manera específica:

1. Establecer la comprensión que tiene el colectivo docente y la dirección de centros educativos sobre la gestión administrativa, para la concreción de las políticas educativas.

2. Determinar la percepción del colectivo docente sobre la eficiencia de la gestión administrativa, para establecer las limitaciones que presenta en lo local la ejecución de las políticas educativas del MEP.

3. Describir los espacios de participación docente en la gestión administrativa del centro educativo.

4. Identificar estrategias de gestión del centro para enfrentar los cambios en las directrices emanadas por el MEP.

\section{Antecedentes}

Los estudios sobre la gestión son variados y multifactoriales, sin embargo, todos coinciden en la necesidad de una gestión eficiente para el logro de procesos de calidad en los centros educativos. Algunas investigaciones, como la de Palacios (2013), se refieren a la implementación de los sistemas de gestión empresarial al campo educativo y los inconvenientes que estos representan para la dirección de los centros educativos, por la naturaleza de estos, así como por los servicios sociales en el cual se centran sus labores.

Por otro lado, Serrano (2015) plantea una investigación longitudinal de la gestión desde los años sesenta del siglo pasado hasta la actualidad, haciendo énfasis en las necesidades de sus actores sociales 
y la complejidad que los constituye como miembros de la comunidad educativa. Además del análisis a las disposiciones políticas a las cuales deben responder las instituciones educativas, se centra en las dificultades económicas desde las que muchos centros educativos operan.

Otra de las investigaciones que propone el análisis de los procesos de la administración en los centros educativos fue realizada por Aguas, Fernández y Cantillo (2013), centrando el foco en procesos básicos como planeación y organización, y las estrategias más pertinentes para el logro del cumplimiento de los objetivos planteados por el centro educativo. Se hacen aportes hacia la delegación y la división del trabajo como parte de las funciones administrativas básicas que requiere la gestión efectiva de la organización. Asímismo, los investigadores concluyen que para que el proceso administrativo sea eficiente se deben alinear áreas como finanzas, mercadeo, talento humano, entre otras. Es por ello que uno de los requerimientos de los centros educativos es el planteamiento de metas claras que vayan articuladas con los objetivos generales, ya que esto facilita la distribución de tareas y estrategias a desarrollar para el logro de estas.

Por otro lado, Ramón y Medina (2014) presentan los resultados de una investigación que describe y analiza cómo la administración del centro educativo asume la formación de su personal docente para desarrollar las competencias básicas requeridas para sus labores. Una de las conclusiones que comparten es que hay cierta precipitación en las decisiones administrativas, además de la existencia de coherencia entre los servicios que se prestan en el centro y los agentes implicados, así como una ausencia de evaluación sobre la transferencia de los procesos de formación y su desempeño propiamente de las labores docentes.

Desde otro abordaje, pero centrado en la gestión de centros educativos, se encuentran los aportes de López, García, Oliva, Moreta y Bellerín (2016), quienes toman el tema de liderazgo de los directivos escolares en sus actividades diarias, concluyendo que los estilos de liderazgo tienen relación con actitudes y sentimientos en el desempeño de las tareas. Además, que las tareas diarias de los directivos escolares como personas encargadas de los procesos administrativos de la organización se relacionan con aspectos como la enseñanza, la gestión, las relaciones sociales y el desarrollo profesional.

Los aspectos que se pueden abordar desde la gestión de centros educativos son multifactoriales, por ello cada institución puede ser un 
universo de posibilidades de investigación, las características, la forma cómo afronta los desafíos, la gestión de los miembros de la comunidad educativa son particulares a su contexto. Asimismo, intervienen tanto factores endógenos como exógenos, haciendo más complejo y dinámico el proceso de gestión.

\section{Marco teórico}

Para la Dirección de Evaluación de la Calidad (DEC) del MEP (2012), el centro educativo es concebido como una unidad básica de construcción donde participan de manera colaborativa tanto docentes como estudiantes. Además, refieren que es una organización multifuncional destinada a la tarea educativa, dentro de un marco legal que señala diferentes niveles para sus estudiantes, con el fin de que estos puedan tener estímulos para su crecimiento físico, social, emocional e intelectual. Este acercamiento conceptual describe un espacio de interacciones complejas, que necesariamente requiere de procesos de gestión que puedan encauzar los esfuerzos de sus miembros para el logro de objetivos comunes. En este sentido la dirección como responsable directa de la gestión administrativa del centro tiene una tarea ardua en la cual tanto los factores endógenos como los exógenos son importantes en esta dinámica.

Lo anterior devela que el centro educativo depende de una dinámica colaborativa para alcanzar objetivos comunes, a pesar de que la dirección es la responsable directa de los procesos administrativos propiamente dichos, la participación de sus miembros es sustantiva. En este sentido, Covey (1997) menciona que la percepción que se tenga de la gestión será un aspecto relevante, ya que para una organización la credibilidad y la confianza en las personas que lideran procesos son básicas para la efectividad de las metas.

La gestión de centros educativos dista de ser una tarea sencilla, esta está constituida por procesos fundamentales de la administración, entre los que destacan, de acuerdo con Chiavenato (2006), la planificación, organización, dirección y ejecución de las propuestas que se desarrollan en las organizaciones. Básicamente, la administración conlleva procesos cíclicos, los cuales requieren de una gestión oportuna para alcanzar metas de manera eficiente y eficaz. 
En este sentido, Chica (2015) aclara que la gestión educativa busca aplicar los principios generales de la gestión, que han estado presentes en la teoría de la administración y se extrapolan al campo específico de la educación. El objeto de la gestión educativa como disciplina es el estudio de la organización del trabajo en el campo de la educación, por tanto, está influenciada por teorías de la administración, pero además existen otras disciplinas que han permitido enriquecer el análisis, como son "la administración, la filosofía, las ciencias sociales, la psicología, la sociología y la antropología" (Chica, 2015, p. 22).

Por lo anterior, para este estudio la gestión administrativa comprende todos aquellos esfuerzos de la dirección destinados a favorecer procesos de mejoramiento de la calidad de la oferta educativa. Dicho acercamiento tiene relación estrecha con los criterios del Modelo de la Evaluación de la Calidad de la Educación Costarricense (MECEC), el cual menciona que la gestión administrativa se da cuando "los usuarios del sistema diseñan, dirigen, ejecutan y evalúan las acciones que permiten el logro de objetivos educacionales" (MEP, 2012, p. 15). Como se observa, el papel de la dirección en la gestión administrativa de los centros educativos es clave para la coordinación de esfuerzos direccionados al logro de metas conjuntas en el campo educativo.

La gestión administrativa consigue sus logros accionando una dimensión colaborativa. Se parte de que el centro educativo desarrolla una labor social de transformación y, por tanto, depende de la participación de cada miembro de la comunidad educativa para que se puedan direccionar todos los esfuerzos hacia un mismo norte. Según Borre11 y Chavarría (2001), este proceso de gestión centra su atención en funciones como "representar, informar, planificar, coordinar, controlar-evaluar, dirigir, administrar y gestionar recursos y animar" (p. 13). En este mismo sentido, Molano (2016) complementa estas funciones con la optimización de procesos pedagógicos, directivos y comunitarios, indicando que la integración de los miembros de la institución, desde una perspectiva participativa, es necesaria para el mejoramiento de la calidad de la educación.

Desde una dimensión colaborativa de la gestión de centros educativos, es sustantivo que los procesos de formación de sus docentes se articulen con las metas y los objetivos que se asumen como parte del mejoramiento de la oferta educativa. El desarrollo profesional de docentes requiere direccionarse hacia temas relevantes en el accionar 
del centro educativo, por lo que las capacitaciones deben gestionarse con propósitos claros para enriquecer las prácticas pedagógicas. Según López, Álvarez y Ocampo (2014) la actualización del colectivo docente es necesaria para analizar, reflexionar y consensuar acuerdos que son indispensables para la mejora continua de los espacios laborales, de vida y culturales. Asímismo, asocian este proceso de formación con el rendimiento académico del estudiantado. Es sabido que los procesos de gestión administrativos conllevan la coordinación del desarrollo profesional, sin embargo, la realidad que los centros educativos experimentan para estos espacios se podría ver afectada por los tiempos que se requieren para concretizarla.

Aunado a lo anterior, han surgido alternativas que responden a las necesidades actuales como la formación en línea. En este sentido, Cenich y Santos (2015) explican que esta alternativa puede ser una posibilidad viable para el grupo de docentes, ya que les puede ayudar a realizar análisis crítico-reflexivos de sus propias prácticas. Además, aportan que dependiendo de cómo se organice el curso, este puede proporcionar aprendizajes significativos y colaborativos.

Otro de los ejes que dinamiza el análisis de este estudio es el liderazgo. Según Bolívar, López y Murillo (2013), "El liderazgo educativo es percibido como un elemento clave para generar mejora desde abajo, creando condiciones institucionales que hagan más eficaces las organizaciones escolares" (p. 1). Sin embargo, el autor Botía (2013) aporta otro elemento a la discusión del liderazgo para las organizaciones educativas y expresa que, para la gestión efectiva de los centros educativos, se requiere de un liderazgo pedagógico, el cual acciona procesos educativos en relación con el contexto donde se encuentra inmersa la institución. Como se observa, realiza una crítica específica a un estilo de liderazgo burocrático, que vendría a representar una limitación para una gestión eficiente.

Desde esta perspectiva del liderazgo pedagógico, Fernández y Hernández (2013) destacan la importancia de favorecer una cultura inclusiva que favorezca la participación de sus miembros desde la validación de sus principios, percepciones, creencias y valores.

Otro de los puntos que se destacan en la discusión de esta investigación es el cambio, entendiéndolo como la capacidad de transición de una situación a otra, este implica transformaciones de aspectos que se asumen como parte de las rutinas del centro educativo. En estos procesos 
de cambio, el desarrollo de la capacidad de adaptación es fundamental. Para Contreras y Barbosa (2013), la importancia del cambio radica en que este permite la evolución de las organizaciones, la cual debería ser abordada por los líderes de la gestión de manera particular para cada contexto.

En la misma línea, Macluf, Beltrán y Chávez (2015) aportan que la gestión de las organizaciones está caracterizada por su complejidad y dinamismo, acentuándose estas características por la competitividad en que se desarrollan los centros educativos en la actualidad. Sin embargo, estos procesos de cambio, en algunas ocasiones, presentan resistencia, que se asocia principalmente a variables psicológicas de sus miembros.

\section{Metodología del proyecto}

El presente estudio responde a una metodología mixta (Creswell, 2009), ya que se aborda tanto desde un enfoque cuantitativo como cualitativo, y un alcance exploratorio-descriptivo. Específicamente, desde una perspectiva cualitativa y un método fenomenológico se pretende entender "el sentido dado a los fenómenos, descubrir el significado (...)" (Bisquerra, 2014, p. 317) que la dirección tiene sobre la administración en los centros escolares.

Por otro lado, desde un enfoque cuantitativo y con un diseño ex post facto y transversal (Hernández, Fernández y Baptista, 2010), se recoge la percepción del colectivo docente de diferentes centros educativos.

\section{Selección de la muestra y participantes}

En primer lugar, se llevan a cabo entrevistas con seis personas a cargo de la dirección de centros educativos públicos. Como se observa en la tabla 1, corresponde a diferentes ámbitos, concretamente uno de preescolar; tres de primaria y dos de secundaria. Las edades de las personas participantes quedan comprendidas en un rango que oscila entre 41 y 55 años. En todos los casos tienen experiencia en dirección superior a los ocho años y como mínimo tres años de laborar en la institución en el momento de la entrevista. 
Tabla 1. Características de las personas participantes en las entrevistas

\begin{tabular}{|lcccc|}
\hline \multirow{2}{*}{ Sexo } & \multicolumn{2}{c}{ Hombre } & \multicolumn{2}{c|}{ Mujer } \\
\cline { 2 - 5 } & \multicolumn{2}{c}{2} & \multicolumn{2}{c|}{4} \\
\hline \multirow{2}{*}{ Ámbito educativo } & \multicolumn{2}{c}{ Preescolar } & Primaria & Secundaria \\
\hline Edad (años) & 41 & 55 & 47,0 & 4,01 \\
\hline $\begin{array}{l}\text { Mínimo } \\
\begin{array}{l}\text { Experiencia en dirección } \\
\text { (años) }\end{array}\end{array}$ & 8 & 15 & 10.3 & 2,40 \\
\hline $\begin{array}{l}\text { Años laborados en la } \\
\text { institución actual }\end{array}$ & 3 & 9 & 6.2 & 4,60 \\
\hline
\end{tabular}

Fuente: Elaboración propia.

Teniendo en cuenta el carácter exploratorio del estudio, la muestra se selecciona de manera no probabilística. En un primer momento se obtienen 246 instrumentos, y con el objetivo de facilitar el análisis, se fijan tres restricciones muestrales: a) tener al menos un año de laborar en el centro educativo en el momento de la recogida de datos; b) tener al menos dos años laborados como experiencia docente $y, c)$ cumplimentar al menos el $75 \%$ del instrumento.

Después de la aplicación de las restricciones, la muestra queda conformada por 192 docentes $(\mathrm{n}=196)$ de diez centros educativos de la Gran Área Metropolitana (Costa Rica) que cumplen con los siguientes criterios: a) de los diferentes niveles del sistema público costarricense: preescolar, primaria y secundaria; b) de diferentes ámbitos: rural y urbano, cabe destacar que dos de los centros se ubican en zonas de atención prioritaria.

Aspectos como la representatividad quedan de manifiesto en la figura 1, donde se observa la cantidad de los centros educativos públicos del país en comparación con la muestra obtenida y donde se aprecia la similitud de los porcentajes tanto poblacionales como muestrales en cada ámbito. 
Figura 1. Frecuencias relativas de la población y de la muestra del estudio

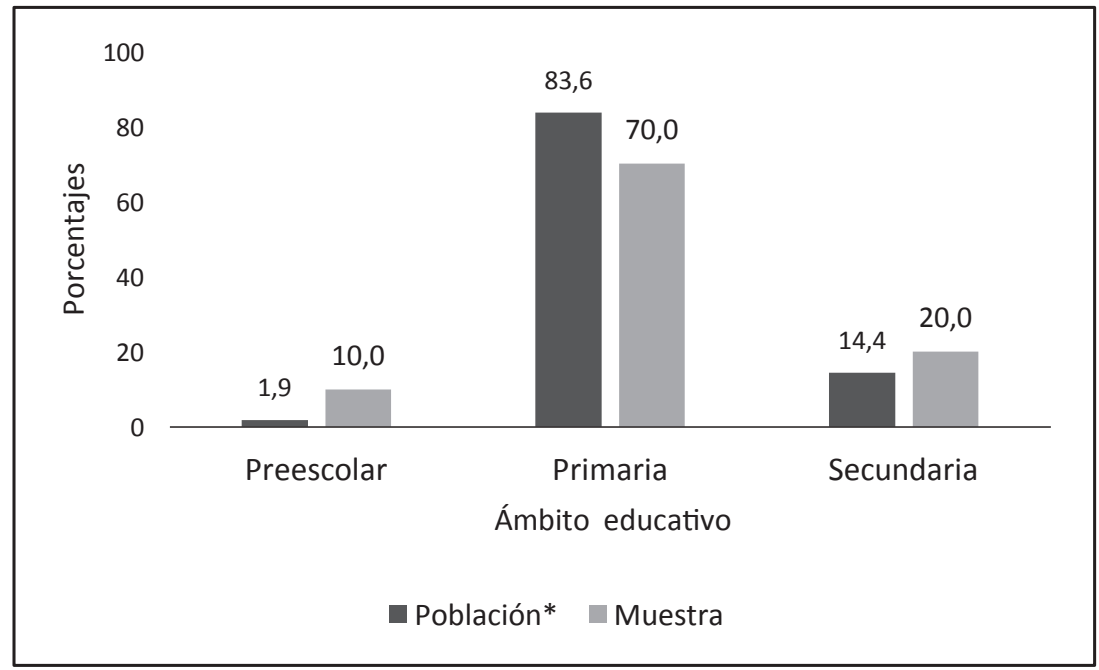

Fuente: Creación propia con indicadores del MEP (2016).

* Los datos de la población corresponden al año 2015.

La muestra queda compuesta por 154 mujeres (80.2\%) y 38 hombres (19.8\%). La edad del colectivo docente oscila entre un rango desde los 27 hasta los 65 años $\left(\mathrm{Md}=38 ; \overline{\mathrm{X}}_{=} 43.2 ; \sigma=8.6\right)$. En cuanto a la distribución por grado académico que poseen, 31 (16.1\%) tienen bachillerato, 134 (69.8\%) cuentan con licenciatura, los restantes $25(13 \%)$ poseen una maestría y solamente 2 personas $(1.0 \%)$ indican tener un doctorado. En la figura 2 se pueden observar los datos comparados por grado académico y nivel. 
Figura 2. Grado académico y nivel donde labora el colectivo docente

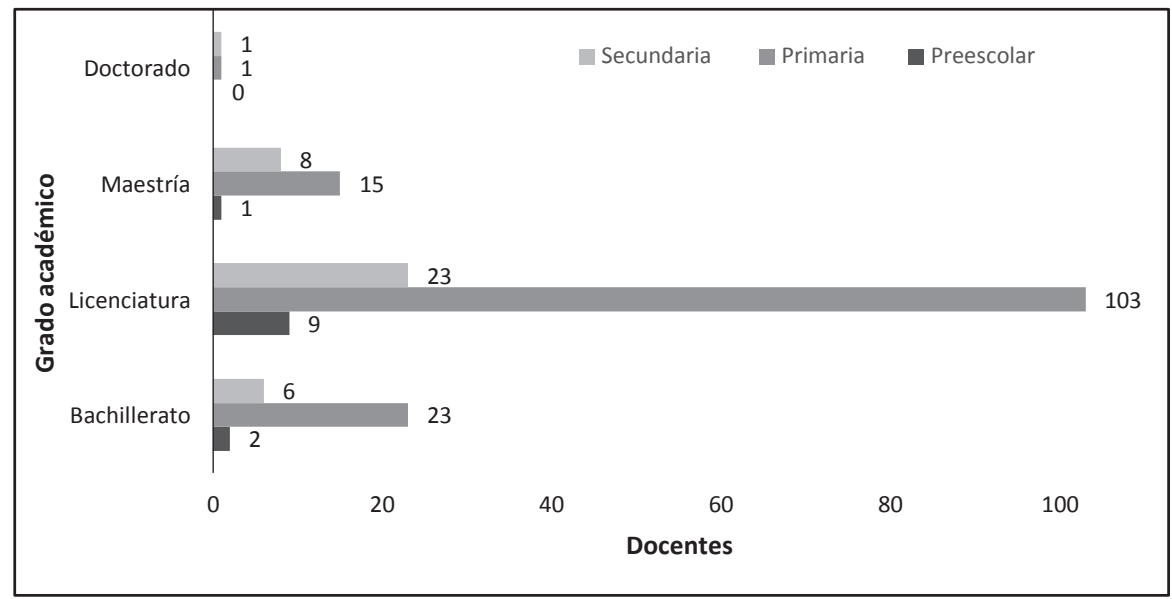

Fuente: Elaboración propia.

En cuanto a las áreas de formación del colectivo docente, la mayoría, $132(69 \%)$ pertenecen a Educación; 18 (10\%) a Ciencias Naturales; $16(8 \%)$ a Ciencias Sociales; 14 (7\%) al área de idiomas y, finalmente, $12(6 \%)$ se ubican en otras áreas distintas a las mencionadas.

El colectivo docente oscila entre los 2 y los 49 años de laborar en educación ( $\overline{\mathrm{X}}=16.3 ; \sigma=8.2)$, y concretamente, entre 2 y 29 años de trabajar en la institución actual. La condición laboral de la muestra es de 174 propietarios $(76.6 \%)$ y 45 interinos $(23.4 \%)$.

\section{Técnicas de recolección de datos}

Partiendo de supuestos teóricos y prácticos, se realiza un estudio mediante la técnica de encuesta (Bisquerra, 2014), en este se utilizan dos instrumentos para la recolección de datos, que se describen a continuación.

En una primera instancia se recolecta información de la dirección de centros educativos, para lo que se utiliza una entrevista semiestructurada, basada en un protocolo previamente elaborado y que es sometido a la validación por juicio de expertos.

De forma paralela se elabora un cuestionario ad hoc, que se aplica a docentes según la descripción de la muestra realizada con anterioridad. 
La estructura queda reflejada en la tabla 2, donde se observa que el instrumento queda compuesto por cuatro bloques y un total de 85 ítems.

Tabla 2. Estructura del cuestionario utilizado para la recogida de datos

\begin{tabular}{|l|c|}
\hline \multicolumn{1}{|c|}{ Bloques } & Cantidad de preguntas \\
\hline 1. Aspectos generales & 12 \\
\hline 2. Gestión curricular & 19 \\
\hline 3. Procesos pedagógicos y de aula & 30 \\
\hline 4. Gestión administrativa & 24 \\
\hline TOTAL & 85 \\
\hline
\end{tabular}

Fuente: Elaboración propia.

El bloque de gestión administrativa, el cual se analiza en el presente estudio, queda compuesto por dos escalas tipo Likert, ambas con cinco opciones de respuesta desde muy de acuerdo (5) hasta muy en desacuerdo (1) la primera de ellas y, una segunda desde nunca (1) hasta siempre (5). Además de dos preguntas, una de ellas cerrada y dicotómica, y la otra de carácter abierto.

Para la validación del cuestionario, se tiene en cuenta el procedimiento que proponen Cohen y Manion (1990), donde se genera una batería de preguntas por parte del equipo investigador y se someten a juicio de expertos. De la devolución se obtiene el primer borrador, con el cual se lleva a cabo un pilotaje con 15 personas con características similares a las de la muestra, posteriormente se realizan los análisis de correlación y de consistencia interna.

Se realiza la prueba de correlación de Pearson entre el componente de gestión administrativa y la escala total, y como se observa en la tabla 3 , existe una fuerte correlación $[\mathrm{r}=.568, \mathrm{n}=196, \mathrm{p}<.000]$. 
Tabla 3. Correlación de Pearson entre la escala total y el componente de gestión administrativa

\begin{tabular}{|l|l|l|r|}
\hline \multicolumn{2}{|l|}{} & $\begin{array}{l}\text { Escala } \\
\text { Total }\end{array}$ & $\begin{array}{l}\text { Escala gestión } \\
\text { administrativa }\end{array}$ \\
\hline Escala Total & Correlación de Pearson & 1 & $.568^{* *}$ \\
\hline & Sig. (bilateral) & & .000 \\
\hline & $\mathrm{N}$ & 196 & 196 \\
\hline
\end{tabular}

** La correlación es significativa al nivel 0,01 (bilateral).

Fuente: Elaboración propia.

Igualmente, se realiza el cálculo de la consistencia interna, concretamente el Alpha de Cronbach obteniendo, 0.76, superior a 0.7, considerado por la teoría como fiable (Cronbach, 1951), por lo que se puede determinar que la escala utilizada es certera.

\section{Presentación de resultados}

La presentación de resultados se realiza de tal forma que respondan a los objetivos planteados. Se presentan de manera simultánea, tanto la información recabada desde la dirección de centros educativos a través de entrevistas, como la obtenida con el cuestionario aplicado al colectivo docente.

La comprensión que tienen las personas encargadas de la dirección de centros educativos sobre la gestión administrativa, como se observa en la tabla 4, devela una perspectiva general en la cual se aprecian algunos aspectos que constituyen la función administrativa.

\section{Tabla 4. Comprensión de la gestión administrativa por parte de la dirección}

\begin{tabular}{|c|l|}
\hline Informante & \multicolumn{1}{c|}{ Comprensión de la gestión educativa } \\
\hline $\mathbf{1}$ & $\begin{array}{l}\text { "Implica las acciones que el centro educativo pueda realizar } \\
\text { para brindar un buen servicio". }\end{array}$ \\
\hline $\mathbf{2}$ & $\begin{array}{l}\text { "Todas aquellas acciones que van a favorecer la buena marcha } \\
\text { de la institución". }\end{array}$ \\
\hline $\mathbf{5}$ & $\begin{array}{l}\text { "Aborda las situaciones con padres, madres y estudiantes, para } \\
\text { el desarrollo del currículo. Interesa la mediación docente, por } \\
\text { lo que se visitan aulas". }\end{array}$ \\
\hline
\end{tabular}

Fuente: Elaboración propia. 
Por otro lado, en la figura 3 se puede apreciar como la mayoría del colectivo docente indica estar muy de acuerdo o de acuerdo con los diferentes aspectos que componen la gestión administrativa, específicamente con la coordinación de personal (80.2\%), la coordinación con organismos de apoyo (77\%), así como con el manejo presupuestario, y coordinación, diseño y evaluación de elementos curriculares dentro de la oferta educativa (en torno a 73\%). Cabe resaltar que alrededor de $27 \%$ (45 docentes) indica no saberlo o estar en desacuerdo con los aspectos que componen la gestión administrativa.

Figura 3. Percepción docente sobre aspectos que comprende la gestión administrativa

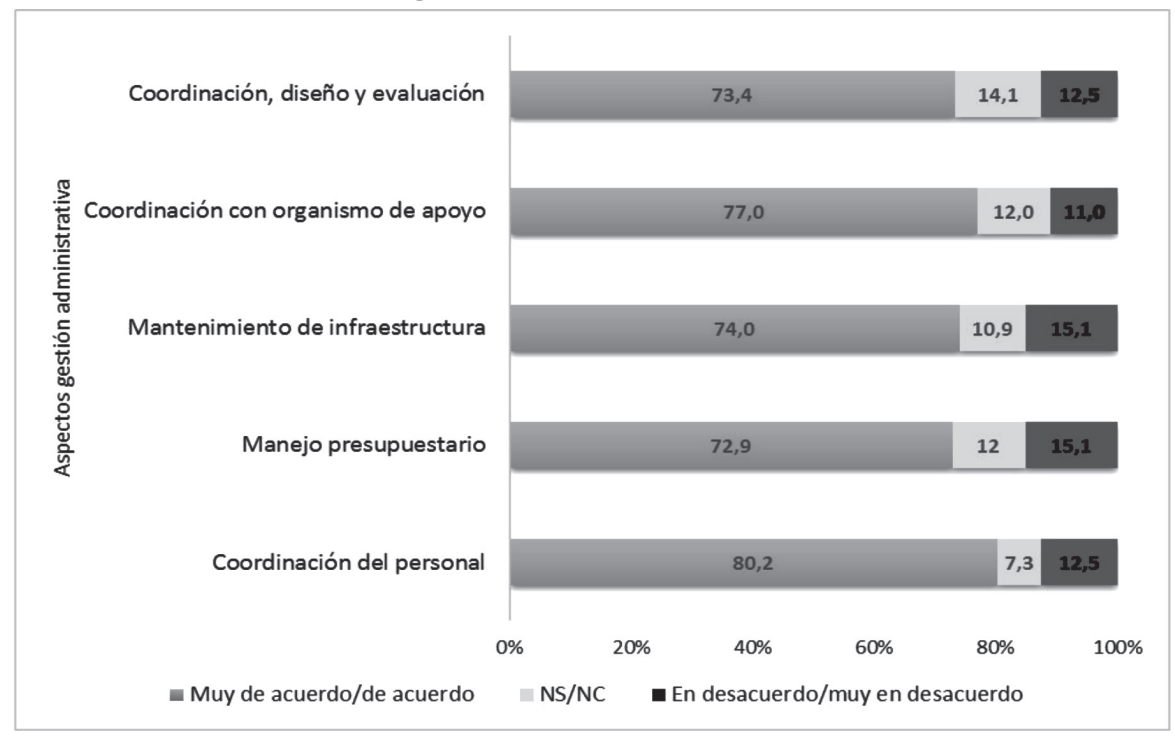

Fuente: Elaboración propia.

En cuanto a la eficiencia sobre algunos aspectos de la gestión administrativa desde la percepción docente (ver figura 4), el colectivo indica estar muy de acuerdo o de acuerdo con como la actualización (64.1\%); procesos de inducción (59.4\%) y el logro de objetivos institucionales (54.2\%). La mitad de la muestra (50\%) indica estar de acuerdo con aspecto como el liderazgo, la relación entre la institución y la comunidad, la participación en el Plan Institucional y la posesión de autonomía para el cumplimiento de los programas establecidos por 
el MEP, aunque cabe resaltar que estos dos últimos aspectos presentan una dicotomía, ya que el otro 50\% aproximadamente manifiesta lo contrario. Cabe resaltar que la gran mayoría (60.1\%) está en desacuerdo o muy en desacuerdo con el hecho de que las directrices emanadas por el MEP responden a las necesidades del contexto donde están inmersos los centros educativos; la infraestructura es otro elemento que el colectivo considera poco eficiente ya que el $48.2 \%$ no está de acuerdo, igualmente los recursos presupuestarios destinados por el Estado para el apoyo a la docencia parecen ser insuficientes (47\%).

Figura 4. Eficiencia sobre aspectos de la gestión administrativa desde la percepción docente

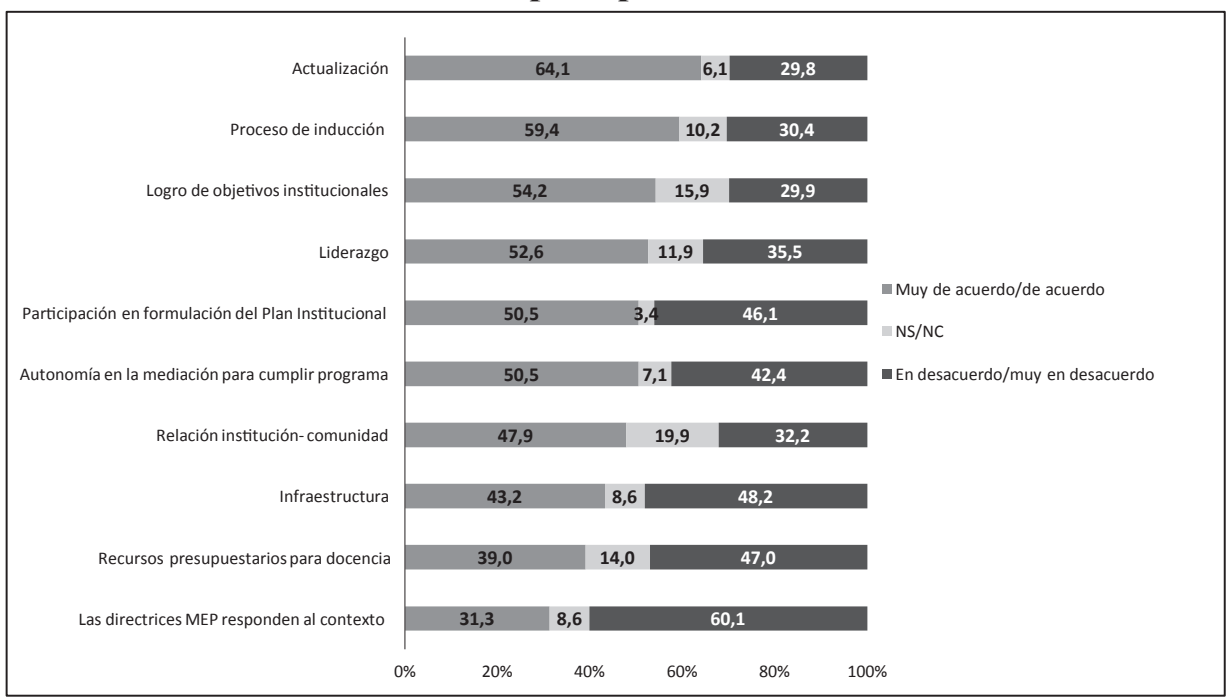

Fuente: Elaboración propia.

A continuación, se indican las respuestas de la dirección en relación con la participación docente en la elaboración de planes y proyectos para el centro educativo. Se observa (tabla 5) que la participación del colectivo docente ocupa un lugar relevante para la gestión administrativa. 
Tabla 5. Participación docente desde la percepción de la dirección

\begin{tabular}{|c|l|}
\hline Informante & \multicolumn{1}{c|}{$\begin{array}{c}\text { Participación en la elaboración de planes y proyectos para el } \\
\text { centro educativo }\end{array}$} \\
\hline $\mathbf{2}$ & $\begin{array}{l}\text { "Todos, es decisión de todo el personal, ya que están ubicados } \\
\text { en diferentes comités y deben hacer sus planes de trabajo". }\end{array}$ \\
\hline $\mathbf{3}$ & "Participa todo el colectivo de docentes". \\
\hline $\mathbf{6}$ & $\begin{array}{l}\text { "Básicamente le corresponde a la administración, pero se pasa } \\
\text { un instrumento a los compañeros para saber sus inquietudes y } \\
\text { sugerencias para tenerlos en cuenta". }\end{array}$ \\
\hline
\end{tabular}

Fuente: Elaboración propia.

Según la muestra de la investigación correspondiente al colectivo docente, la participación en comités de apoyo es de 97 docentes $(50.5 \%)$ y no participan 95 (49.5\%). En la figura 5, se observan los diferentes espacios donde mayor participación tienen, siendo el comité de apoyo educativo (22\%) y las ferias científicas y de artes $(20.7 \%)$ o que mayor participación manifiestan, seguidos de otros comités $(14.6 \%)$ relacionados con deportes, tecnologías, nutrición, etc., además de los comités de evaluación (13.4\%) y de educación (12.2\%).

\section{Figura 5. Participación docente en diferentes comités de apoyo}

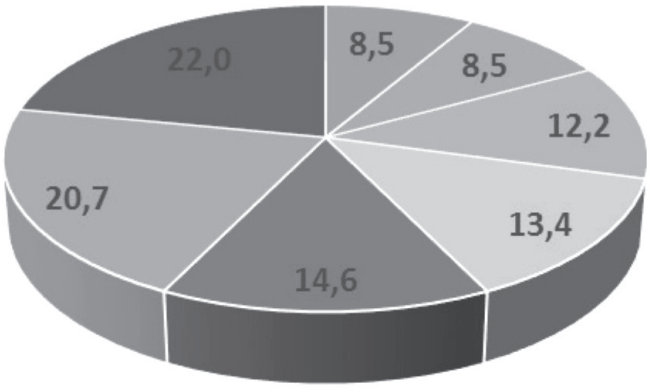

- Bandera azul

= Cultura de Paz

= Calidad de educación

- Evaluación

- Otros

- Feria científica y FEA

- Apoyo educativo

Fuente: Elaboración propia. 
En la tabla 6, se observan los principales mecanismos que el personal encargado de la dirección indica que se llevan a cabo para informar al colectivo docente sobre las nuevas disposiciones del MEP. Como se aprecia, las reuniones de personal y las circulares son medios asiduos para dicha comunicación.

Tabla 6. Mecanismos de los centros para comunicar las nuevas disposiciones del MEP

\begin{tabular}{|c|l|}
\hline Informante & \multicolumn{1}{|c|}{$\begin{array}{c}\text { Mecanismos que tiene el centro para comunicar las nuevas } \\
\text { disposiciones del MEP }\end{array}$} \\
\hline $\mathbf{1}$ & $\begin{array}{l}\text { "Por medio de circulares, reunión de personal, de forma digital } \\
\text { por correo electrónico". }\end{array}$ \\
\hline $\mathbf{5}$ & "Reuniones y circulares en mayor medida". \\
\hline $\mathbf{6}$ & $\begin{array}{l}\text { "Siempre con reuniones de personal, ya que la comunicación } \\
\text { escrita no es lo que uno espera, y así se pueden aclarar dudas, } \\
\text { aunque aspectos poco profundos como cambios de fechas etc., } \\
\text { se hace por circular o un chat". }\end{array}$ \\
\hline
\end{tabular}

Fuente: Elaboración propia.

En cuanto a la percepción docente, se puede ver (figura 6) que las nuevas directrices y normativas del MEP son comunicadas siempre o casi siempre a través de circulares $(67.3 \%)$, seguidas de las reuniones de personal (63.4\%); y en menor medida se solventan a través de la iniciativa propia del colectivo docente (48.4\%) y talleres (44.8\%). Cabe resaltar que un gran porcentaje del colectivo indica que nunca o casi nunca es informado a través de las personas asesoras del MEP (41.2\%) o bien de la página web del MEP (30.2\%). Igualmente es importante destacar, que en todas las categorías un alto porcentaje señala ser informado algunas veces. 
Figura 6. Procedencia de la información de las nuevas directrices y normativa MEP según la percepción docente

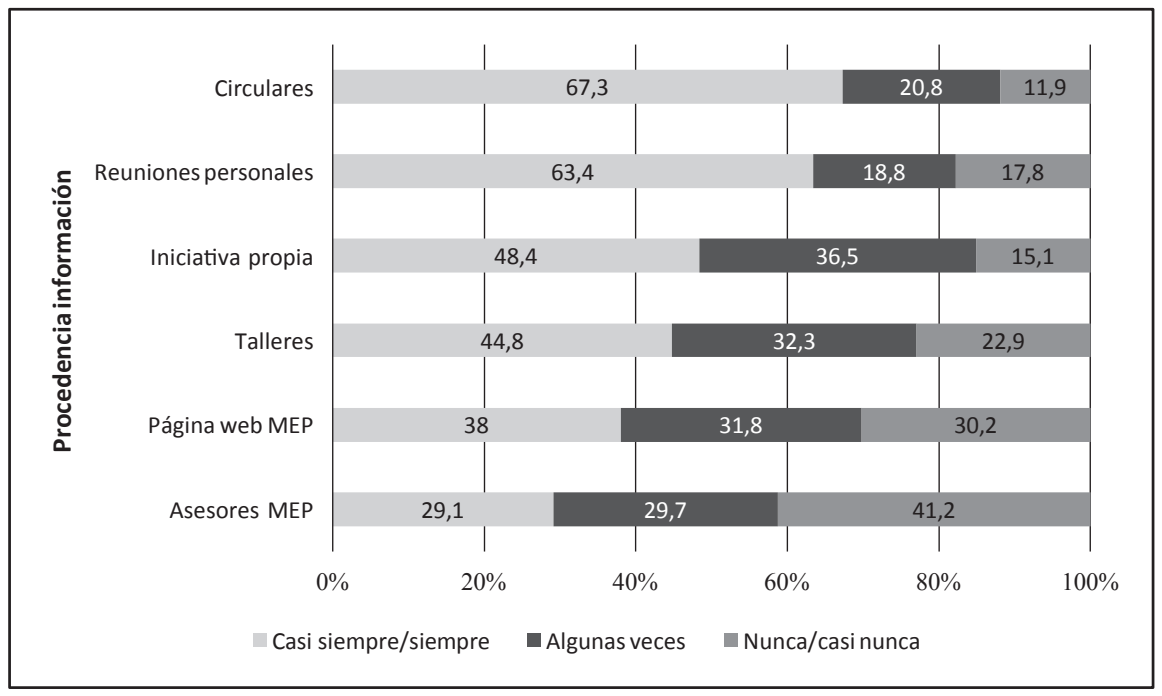

Fuente: Elaboración propia.

\section{Discusión y conclusiones}

Las organizaciones educativas son espacios de construcción social donde se requiere de un trabajo colaborativo, que permita alcanzar metas y objetivos comunes. La participación de sus miembros es sustantiva en estos procesos, ya que de ellos depende la concreción de una acción efectiva. Por lo anterior, se considera relevante la comprensión que el colectivo docente y la dirección tengan sobre las funciones de la gestión administrativa, especialmente en lo que se refiere a las políticas sobre gestión generadas por el MEP y que deben concretarse en lo local, a partir del liderazgo que despliegue la figura de la dirección del centro.

Cabe resaltar la importancia del presente estudio, donde se plantea el análisis de la percepción del colectivo docente y de la dirección sobre aspectos relacionados con la gestión administrativa de los centros educativos a partir de las disposiciones (políticas educativas) del MEP, para incidir en los factores limitantes desde el proceso de formación superior y responder desde la constante adecuación y revisión de procesos de formación en educación superior a las demandas sociales y educativas. 
Como se desprende de los resultados por grupos etáreos y de las calidades académicas del grupo de educadores participantes, la mayoría (69.8\%) posee el título de licenciatura y cuenta con experiencia laboral media de nueve años en instituciones educativas públicas, de ahí que se puede afirmar, que son profesionales que han tenido la oportunidad de experimentar y entender las dinámicas internas de la gestión de un centro educativo, que conocen las orientaciones emanadas del MEP y cómo se pueden concretizar finalmente en el aula.

Con respecto al grupo de directivos educativos consultados, conformado por seis profesionales que han tenido la experiencia laboral de al menos diez años de antigüedad en este cargo, se podría partir del supuesto que son administradores que saben y han tenido la oportunidad de poner en marcha las disposiciones ejecutivas curriculares y administrativas que vienen configurando las políticas educativas en los últimos diez años de este país.

Los resultados de este estudio muestran que el colectivo docente expresa tener amplia comprensión de que aspectos como la coordinación de personal y el trabajo con los organismos de apoyo, son acciones que deben liderarse desde la gestión administrativa. Asímismo, reconoce que las funciones de manejo presupuestario, coordinación, diseño y evaluación de aspectos curriculares son actividades inherentes a la gestión administrativa. Dichos resultados se ajustan a los referentes conceptuales aportados por Borrell y Chavarría (2001) y Aguas et al. (2013), los cuales refieren a que las funciones de la gestión administrativa se centran en procesos de planeación y organización, así como en el establecimiento de estrategias que faciliten el logro de las metas y los objetivos planteados por el centro educativo.

La importancia de reconocer a quién corresponde asumir diferentes roles y tareas en un centro educativo es de vital relevancia, ya que es en este aspecto donde radica la legitimidad que adquiere el liderazgo que la dirección puede y debe desarrollar con el colectivo docente. Dicha legitimidad también repercute en la participación del trabajo voluntario que aportan los padres y las madres de familia que colaboran en las Juntas de Educación y las Juntas de Administración para la concreción de las orientaciones del MEP. Lo anterior se considera sustantivo en la gestión administrativa del centro educativo, ya que estos organismos de apoyo se constituyen en invaluables colaboradores de la gestión. 
En cuanto a la percepción de quién debe ejecutar las disposiciones y orientaciones que concretizan las políticas educativas en los ámbitos locales, tanto el colectivo docente como la dirección destacan la importancia de que exista la división de tareas en la gestión. Además, existe consenso sobre el rol de liderazgo que se adjudica a la dirección, destacando procesos relacionados con el manejo presupuestario la coordinación con el personal y organismos de apoyo, entendiendo éstos como las Juntas de Educación y de Administración, los comités de apoyo establecidos en el aula y conformados por padres y madres de familia, entre otros.

Sobre esto último, conviene resaltar que existe un $27 \%$ del colectivo docente que manifiesta desconocimiento sobre cuáles son las funciones asignadas a la gestión administrativa. Pareciera que este aspecto constituye una limitante para comprender las acciones administrativas y curriculares que definen el rol de la dirección con miras a la articulación de esfuerzos y tareas que lleven a resultados efectivos sobre las metas y los objetivos planteados en la organización.

En este sentido, se coincide con lo expuesto por López et al. (2016), quienes mencionan que el papel de la dirección escolar como líder de los centros educativos, debe prestar especial interés a las actitudes y los sentimientos del colectivo docente, ya que ello va a tener implicaciones directas con su desempeño, concordando con lo expresado por Serrano (2015). El papel de la dirección implica también, la gestión de las relaciones sociales y el desarrollo profesional, tal y como lo señala un directivo al indicar que se encarga de "potenciar los perfiles que llegan a la escuela, motivarlos y enamorarlos para que quieran trabajar aquí".

La percepción del colectivo docente sobre la eficiencia de la gestión administrativa revela la existencia de una valoración positiva sobre aspectos como procesos de inducción y actualización y el logro de objetivos propuestos para el centro educativo. En este sentido, cabe resaltar que existe una amplia oferta para actualización por parte del MEP, lo que vendría a respaldar esta percepción.

Sin embargo, cerca de la mitad de los encuestados perciben poca eficiencia en aspectos como el liderazgo, la relación entre la institución y la comunidad, la participación en la formulación del Plan Institucional, así como el ejercicio de autonomía en la ejecución áulica de los programas establecidos por el MEP, de ahí se puede interpretar que la 
eficiencia de la gestión en aspectos sustantivos como los mencionados no es valorada de manera positiva por los docentes. En relación con lo anterior, este podría ser considerado como un importante factor que limita la comprensión sobre algunas de las directrices del MEP, con respecto a las nuevas líneas de acción para la implementación de programas y participación docente.

Por otro lado, la valoración que realizan los profesionales de la dirección de centros es opuesta a lo que indica el colectivo docente, ya que aducen que la gestión administrativa "es eficiente y que aspectos como el presupuesto destinado para las labores docentes por parte del Estado es suficiente para solventar las necesidades que se generan en los procesos pedagógicos", otro informante de la dirección señala que "tenemos un $98 \%$ de resultados en los indicadores de logro, este centro está entre los mejores de la zona". Además, dicen estar "cómodos" con la imagen que proyectan como líderes de la organización. Siguiendo con esta línea de análisis, Covey (1997) menciona que la percepción que se tenga de la gestión es un punto relevante, ya que en el centro educativo la credibilidad y la confianza en las personas que lideran la organización son básicas para la efectividad de los procesos.

Asímismo, las directrices emanadas por el MEP, para dar respuesta a las necesidades del contexto educativo en el cual están inmersos los centros educativos, presentan un $60.1 \%$ de desaprobación desde la perspectiva docente. Desde la mirada del equipo investigador, la relación y coordinación desde el centro educativo con la comunidad y la atención a las necesidades del contexto son de vital importancia. De tal manera que, si la percepción del colectivo docente es negativa en este aspecto, podría considerarse como uno de los factores que limitan la concreción de las políticas educativas en el ámbito local, desde la gestión administrativa. En este sentido, Aguas et al. (2013) refieren que existen algunos procesos básicos como planeación y organización, que pueden tener un vínculo directo con la efectividad de logros planteados por el centro educativo. Los investigadores mencionados concluyen que una gestión eficiente debe alinear áreas como finanzas, mercadeo, talento humano, entre otras.

Por otro lado, aspectos como la infraestructura y el manejo de los recursos presupuestarios destinados a satisfacer las necesidades de la labor docente son valorados como poco eficientes por la mitad de la muestra aproximadamente, asunto que queda respaldado por un informante de la dirección al indicar que, "necesitamos quitar el hacinamiento 
ya que esta escuela es muy perseguida en cuanto a matrícula". Con respecto al tema de manejo presupuestario otro informante señala que "este año hemos ahorrado en mano de obra, pero no es suficiente, se paga mucho de luz, agua y teléfono". Esta afirmación puede relacionarse con las conclusiones de la investigación desarrollada por Serrano (2015), quien aduce que la complejidad de los centros educativos se ve incrementada con los problemas económicos que son una constante de la gestión y que obliga a la dirección a invertir energía y tiempo en la búsqueda de recursos para cubrir los faltantes presupuestarios y garantizar el ágil funcionamiento del centro.

Esta percepción tan diferenciada entre los docentes y la dirección de los centros educativos puede tener una explicación en los aportes de Borrell y Chavarría (2001), al indicar que la dirección tiene funciones de representación, y que debe brindar información, planificar, coordinar, evaluar, dirigir, gestionar recursos y motivar a los miembros de la organización, es comprensible que la dirección considere que estas funciones sean eficientes, partiendo de los esfuerzos que debe realizar para obtener nuevos ingresos que garanticen el fluido funcionamiento del plantel educativo.

En este mismo sentido, Molano (2016) señala que las funciones de la dirección deben contemplar otros procesos pedagógicos, directivos y comunitarios, que constituyen aspectos relevantes para que el centro educativo pueda responder de manera efectiva a las demandas de los contextos donde se encuentran inmersos las instituciones educativas. Asímismo, este autor menciona que el trabajo colaborativo de los miembros del centro educativo es necesario para el mejoramiento de la calidad de la educación.

La participación del colectivo docente es un aspecto que cobra relevancia en los centros educativos, ya que como lo indica el MEP-MECEC (2012) el centro educativo es concebido como una unidad básica de construcción donde participan de manera colaborativa tanto docentes como estudiantes. En este sentido, llama la atención que un informante de la dirección manifiesta que "las acciones que se implementan en el centro educativo son conocidas por el colectivo docente, ya que tienen participación en la elaboración de planes y proyectos que se desean implementar en la organización".

Sin embargo, desde la perspectiva docente solo la mitad de los encuestados dice participar activamente en algunos comités que se 
accionan en el centro educativo y que tienen vínculo directo con las metas y los objetivos que se desean alcanzar. Asimismo, se puede observar que la otra mitad de docentes señalan no participar de ningunos de los comités que se gestionan en el centro educativo. Quizá el punto más importante de tomar en cuenta es que, una situación así, plantea un significativo dilema a la dirección en la búsqueda y concreción de las políticas educativas en su centro, puesto que solo la mitad de sus colaboradores manifiestan estar involucrados en el proyecto de centro. En esta línea de ideas, Fernández y Hernández (2013) destacan la importancia de favorecer una cultura inclusiva que favorezca la participación activa de sus miembros desde la validación de sus principios, percepciones, creencias y valores.

En cuanto a la participación del colectivo docente en diferentes actividades del centro educativo, el comité que tiene como objetivo brindar apoyo al estudiantado para mejorar su rendimiento académico es uno de los que con mayor frecuencia se menciona, seguido de los comités que involucran ferias que favorecen conocimientos científicos y artísticos.

De las respuestas sobre participación en los centros educativos, se puede inferir que existe meridiana claridad sobre la valía de no asumir la participación únicamente en términos de obligatoriedad (las tareas y funciones inherentes a los cargos), sino que es importante dimensionarla hacia un trabajo colaborativo que genera procesos identitarios, sentido de pertenecía y, por consiguiente, un aporte de cada uno de sus miembros desde sus espacios individuales, direccionados hacia metas u objetivos comunes.

En lo que se refiere a las estrategias de gestión del centro para enfrentar los cambios en las directrices emanadas por el MEP, un importante instrumento de información y comunicación por parte de la dirección lo constituyen las reuniones de personal y las circulares escritas en las cuales se dan a conocer las nuevas directrices y normativas dispuestas por el MEP, así lo indica la mayoría del colectivo docente. En relación con el papel de los asesores del Ministerio, el colectivo docente no lo considera un ente de apoyo significativo para la concreción de políticas curriculares, ya que casi la mitad manifiesta no recibir información proveniente de estos profesionales.

Un importante dato que cabe destacar, es que menos de la mitad de las personas encuestadas indican basarse en la iniciativa propia para adquirir la información, lo que incluye la utilización de la página web 
del MEP, aspecto que cobra relevancia, si se tiene en cuenta que esta es considerada como una alternativa viable de actualización. Desde esta perspectiva de análisis, Cenich y Santos (2015) explican que las alternativas de información virtuales están abriéndose espacio como una opción factible para algunos docentes; pero al menos entre los grupos encuestados esta opción aún no se convierte en viable. Se trata entonces, de tener en cuenta esta situación para potenciar este valioso recurso a la hora de dar respuesta desde la educación superior a las limitantes que se establecen como barreras para la comprensión de las políticas educativas identificadas en el presente estudio.

Los procesos de gestión en la búsqueda de alternativas para afrontar los cambios en las políticas educativas se asocian a los espacios de formación del colectivo docente, ya que algunos de los cambios planteados en dichas políticas conllevan nuevos acercamientos conceptuales y metodológicos. De aquí se justifica la necesidad de que los espacios de formación se constituyan en una de las funciones sustantivas de la gestión administrativa, como lo mencionan López, Álvarez y Ocampo (2014), al señalar que la actualización del profesorado es necesaria porque permite compartir espacios de análisis, reflexión y generar consensos sobre sus propias prácticas. Estos espacios enriquecen la vida laboral, cultural y social de las organizaciones, dando como resultado procesos de mejora para el centro educativo.

Cabe destacar que la función de la gestión administrativa de afrontar los cambios que se establecen como oficiales desde el MEP, tiene en la práctica una limitación importante, pues no se cuenta con un espacio de tiempo destinado para esto. Tal como indica uno de los entrevistados de la dirección, "se hace necesario implementar estrategias apoyadas por las autoridades centrales del Ministerio de Educación, para brindar espacios formales a los centros educativos en los cuales se brinde información y explicación de los planes y proyectos educativos, de las nuevas disposiciones".

Para finalizar, y como se ha señalado desde el inicio, se reitera la importancia de articular los procesos de formación docente y administrativa, con las metas y los objetivos asumidos como parte del mejoramiento de la oferta educativa desde las políticas educativas, teniendo claro que las prácticas pedagógicas que se establezcan en el centro educativo deben tener total coherencia con los espacios de formación del colectivo docente. 


\section{Referencias}

Aguas, C. A. G., Fernández, E. D. C. G. y Cantillo, L. P. H. (2013). Procesos de planeación y organización en la institución educativa técnica industrial Antonio Prieto. Jornada matinal de la ciudad de Sincelejo en 2011-029. Revista Pensamiento Gerencial. Recuperado de http://revistas.unisucre.edu.co/index.php/rpg/article/view/82

Bisquerra, R. (coord.). (2014). Metodología de la investigación educativa. Madrid, España: Editorial La Muralla.

Bolívar, A., López, J. y Murillo, F. J. (2013). Liderazgo en las instituciones educativas. Una revisión de líneas de investigación. Recuperado de https://idus.us.es/xmlui/handle/11441/33743

Borrell, E. y Chavarría, X. (2001). La planificación y autoevaluación del trabajo de los directivos de centros docentes. Monografías de Escuela Española. Barcelona, España: CISS-PRAXIS-Educación.

Botía, A. B. (2013). Cambio y liderazgo educativo en tiempos de crisis. In Organización y gestión educativa. Revista del Fórum Europeo de Administradores de la Educación (Vol. 21, No. 4, pp. 14-17). Ciss Praxis. Recuperado de https://ialnet.unirioja.es/servlet/ articulo?codigo $=4298483$

Cenich, G. y Santos, G. (2015). Aprendizaje significativo y colaborativo en un curso online de formación docente. Revista electrónica de investigación en educación en ciencias, 4(2). Recuperado de http://ppct.caicyt.gov.ar/index.php/reiec/article/view/7386

Chiavenato, I. (2006). Introducción a la Teoría General de la Administración. Sétima edición. México: Mc Graw-Hill.

Chica, C. A. B. (2015). Cinco tendencias de la gestión educativa. Revista Politécnica, 3(5), 19-31. Recuperado de http://www.plagioplus.com/index.php/pol/article/view/71

Cohen, L. y Manion, L. (1990). Métodos de investigación educativa. Madrid: La Muralla.

Contreras, F. y Barbosa, D. (2013). Del liderazgo transaccional al liderazgo transformacional: implicaciones para el cambio organizacional. Revista virtual universidad católica del norte, 2(39), 152-164. Recuperado de: http://revistavirtual.ucn.edu.co/index. php/RevistaUCN/article/view/433

Covey, S. (1997). El liderazgo centrado en principios. España: PAIDOS. 
Creswell, J. W. (2009). Research Design: qualitative, quantitative, and mixed methods approaches (3rd ed.). London: Sage.

Cronbach, L. J. (1951). Coefficiente alfa and the internal structures of tests [Coeficiente Alfa y estructura interna de los test]. Psychometrika, 16(3), 297-334. Recuperado de http://psych.colorado. edu/ carey/courses/psyc5112/readings/alpha_cronbach.pdf

Fernández, J. M. y Hernández, A. (2013). Liderazgo directivo e inclusión educativa: Estudio de casos. Perfiles educativos, 35(142), 27-41. Recuperado de http://www.scielo.org.mx/scielo. php?script=sci_arttext\&pid=S0185-26982013000400003

Hernández, R, Fernández, C y Baptista, P. (2010). Metodología de la investigación. México, D.F: Mc Graw Hill.

López, A. Y. G., Álvarez, A. G. y Ocampo, H. F. P. (2014). El impacto de la formación docente en el rendimiento académico del estudiante. El caso de la Universidad Autónoma de Nayarit. Inclusión con Responsabilidad-Tomo III, 7. Recuperado de http://s3.amazonaws.com/academia.edu.documents/38880625/ INCLUSION_CON_RESPONSABILIDAD_III.pdf?AWS AccessKeyId=AKIAJ56TQJRTWSMTNPEA\&Expires $=1476913615 \&$ Signature $=$ CKdpcWBjPw6PXeTO3DFxLCC$5 \mathrm{kXQ} \% 3 \mathrm{D} \&$ response-content-disposition $=$ inline $\% 3 \mathrm{~B} \% 20$ filename\%3DInclusion_con_responsabilidad.pdf \#page $=7$

López-Yáñez, J., García-Jiménez, E., Oliva-Rodríguez, N., Moreta-Jurado, B. y Bellerín, A. (2016). El liderazgo escolar a través del análisis de la actividad diaria de los directores. REICE. Revista Iberoamericana sobre Calidad, Eficacia y Cambio en Educación, 12(5). Recuperado de https://revistas.uam.es/index.php/ reice/article/view/2828

Macluf, J. E., Beltrán, L. A. D. y Chávez, R. M. A. (2015). El desarrollo organizacional y la resistencia al cambio en las organizaciones. Ciencia Administrativa, 1, 1-9. Recuperado de http://revistas. uv.mx/index.php/cadmiva/article/view/1604

Ministerio de Educación Pública. (2012) Dirección de Gestión y Evaluación de la Calidad. Glosario de la Educación (1 ${ }^{\mathrm{a}}$ ed). San José, Costa Rica: MEP.

Molano, A. D. (2016). La gestión educativa: Hacia la optimización de la formación docente en la educación superior en Colombia. Sophia, 12(1), 55-70. 
Palacios, J. A. D. (2013). Calidad educativa: un análisis sobre la acomodación de los sistemas de gestión de la calidad empresarial a la valoración en educación. Tendencias pedagógicas, 21, 177-194. Recuperado de https://dialnet.unirioja.es/servlet/ articulo? codigo $=4183206$

Ramón, M. R. R. y Medina, C. P. (2014). La formación en competencias básicas: un reto para la administración. Profesorado: Revista de curriculum y formación del profesorado, 18(2), 245-265. Recuperado de http://www.ugr.es/ recfpro/rev182COL6.pdf

Serrano, J. S. C. S. (2015). Evolución de la Administración y la Gestión Escolar. Tendencias Pedagógicas, 14, 159-168. Recuperado de https://revistas.uam.es/tendenciaspedagogicas/article/ view/1910/0 